\title{
THE EFFECT OF RHEOLOGY WITH GAS BUBBLES ON LINEAR ELASTIC WAVES IN FLUID-SATURATED GRANULAR MEDIA
}

\author{
A.A. ALI and D.V. STRUNIN ${ }^{*}$ \\ Computational Engineering and Science Research Centre \\ Faculty of Health, Engineering and Sciences, University of Southern Queensland \\ Toowoomba, AUSTRALIA \\ E-mail: Dmitry.Strunin@usq.edu.au
}

\author{
A.A. ALI \\ Department of Mathematics, Kirkuk University, Kirkuk, IRAQ
}

\begin{abstract}
Elastic waves in fluid-saturated granular media depend on the grain rheology, which can be complicated by the presence of gas bubbles. We investigated the effect of the bubble dynamics and their role in rheological scheme, on the linear Frenkel-Biot waves of P1 type. For the wave with the bubbles the scheme consists of three segments representing the solid continuum, fluid continuum and bubbles surrounded by the fluid. We derived the Nikolaevskiy-type equation describing the velocity of the solid matrix in the moving reference system. The equation is linearized to yield the decay rate $\lambda$ as a function of the wave number $k$. We compared the $\lambda(k)$-dependence for the cases with and without the bubbles, using typical values of the input mechanical parameters. For both the cases, the $\lambda(k)$ curve lies entirely below zero, which implies a global decay of the wave. We found that the increase of the radius of the bubbles leads to a faster decay, while the increase in the number of the bubbles leads to slower decay of the wave.
\end{abstract}

Key words: Frenkel-Biots waves, bubbles, rheology, porous media.

\section{Introduction}

The fundamentals of the theory of wave propagation in porous elastic solids can be found in $[1,2]$ or, for a more recent review, [3]. In [1, 2] Biot generalised the first principles of linear elasticity and today, most studies in acoustics, geophysical and geological mechanics rely on his theory. Biot also deduced $[4,5]$ the dynamical equations for the wave propagation in poroelastic media. The elastic modulus of the porous matrix with first-order nonlinearity was described in [6]. According to the Frenkel-Biots theory, there are two types of longitudinal waves propagating in a saturated porous medium. The first type is fast and weakly damped (P1-wave), whereas the other type is slow and strongly damped (P2-wave). The slow P-wave has first been observed in a laboratory by Plona [7]. Yang et al. [8] showed that the dispersion of velocity and attenuation of the fast P1-wave are both affected by the viscoelasticity of the medium, but has almost no effect on the slow P2-wave. In addition, they proved that the dominant frequency of the fast P1-wave shifts linearly toward lower frequencies due to the conditions of low permeability and low porosity; this plays a significant role in exploration for gas and oil.

\footnotetext{
${ }^{*}$ To whom correspondence should be addressed
} 
Nikolaevskiy [9] derived a model describing the propagation of nonlinear longitudinal waves in a viscoelastic medium taking into account complicated rheology of grains

$$
\frac{\partial v}{\partial t}+v \frac{\partial v}{\partial x}=\sum_{p=1}^{5} A_{p+1} \frac{\partial^{p+1} v}{\partial x^{p+1}}
$$

where $v$ is the velocity of the solid matrix and the coefficients $A_{p+1}$ are constants linked to mechanical parameters of the system. The terms in Eq.(1.1) account for the effects of non- linearity, dissipation and dispersion. Based on [9] Nikolaevskiy extended [10] the evolution Eq.(1.1) to include the global dissipation

$$
\frac{\partial v}{\partial t}+N v \frac{\partial v}{\partial x}+\varsigma v=\sum_{p=1}^{5} A_{p+1} \frac{\partial^{p+1} v}{\partial x^{p+1}}
$$

where $\varsigma$ and $N$ are constants. Experimental evidence indicated that the existence of gas bubbles in the saturated porous medium changes the characteristics of this medium [11]-[13], acoustic properties and the velocity of the wave. As Nikolaevskiy pointed out [10], in rocks saturated with fluids, the P1-wave is the only observable wave. However, the presence of gas, even in small proportion can affect the wave type [14], so that $\mathrm{P} 2$-wave may be visible.

Dunin et al. studied [11] the effect of gas bubbles on the P1- and P2-waves. They found that the dispersion curve of the P2-wave consists of two branches: a low-frequency branch and a high-frequency branch. In this work, a simple stress-strain relation was used, $\sigma=E e$ (in standard notations). Nikolaevskiy used a much more complicated stress-strain relation that involves higher-order time derivatives of the stress $\sigma$ and strain $e$. This relation is the result of the rheological scheme shown in Fig.1. Eventually, it leads to the higher-order partial differential Eq.(1.1). However, the original rheological scheme [9] does not include gas bubbles. Nikolaevskiy and Strunin [14] pointed out the place in this scheme that the bubbles should take, see Fig.1. In the present work, we aim to include the bubble into the rheological scheme and, based on this, derive the Nikolaevskiy-type Eq.(1.1), where the coefficients $A_{p}$ will depend on the bubble-related parameters.

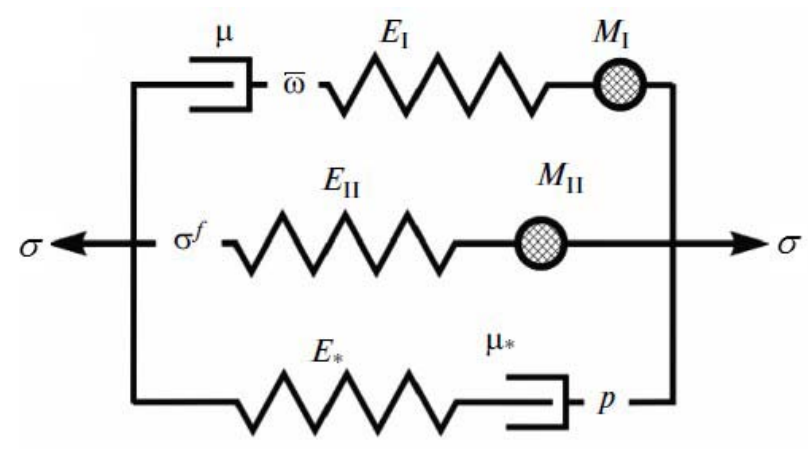

Fig.1. Rheological scheme for the grain. The branch $\varpi$ corresponds to the bubble [14].

\section{Basic equations of one-dimensional dynamics}

\subsection{Conservation of mass and momentum}

For a one-dimensional case the momentum and mass balance equations are 


$$
\begin{aligned}
& \frac{\partial}{\partial t}(1-m) \rho^{(s)} v+\frac{\partial}{\partial x}(1-m) \rho^{(s)} v v=\frac{\partial}{\partial x} \sigma^{(e f)}-(1-m) \frac{\partial p}{\partial x}-I, \\
& \frac{\partial}{\partial t} m \rho^{(f)} u+\frac{\partial}{\partial x} m \rho^{(f)} u u=-m \frac{\partial p}{\partial x}+I, \\
& \frac{\partial}{\partial t}(1-m) \rho^{(s)}+\frac{\partial}{\partial x}(1-m) \rho^{(s)} v=0, \\
& \frac{\partial}{\partial t} m \rho^{(f)}+\frac{\partial}{\partial x} m \rho^{(f)} u=0
\end{aligned}
$$

where the subscripts $s$ and $f$ label the solid and gas-liquid mixture, respectively, $\rho, v$, and $u$ are the corresponding densities and mass velocities, $m$ is the porosity, $\sigma^{(e f)}$ is the effective Terzaghi stress, $p$ is the pore pressure, and $I$ is the interfacial viscous force approximated by

$$
I=\delta m(v-u), \quad \delta=\frac{\mu^{(f)} m}{k}
$$

where $\mu^{(f)}$ is the gas-liquid mixture viscosity and $k$ is the intrinsic permeability.

\subsection{Dynamics of bubbles}

The equation of the dynamics of a bubble [11] has the form

$$
R \frac{\partial^{2}}{\partial t^{2}} R+\frac{3}{2}\left(\frac{\partial}{\partial t} R\right)^{2}+\frac{4 \mu}{\rho^{(L)}}\left(\frac{1}{R}+\frac{m}{4 k} R\right) \frac{\partial}{\partial t} R=\left(p_{g}-p\right) / \rho^{(L)}
$$

where $R$ is the bubble radius, $p$ is the pressure in the liquid, $p_{g}=\left(R_{0} / R\right)^{\chi}$ is the gas pressure inside the bubble (here $\chi=3 \varsigma, \varsigma$ is the adiabatic exponent), $\rho^{(L)}$ is the density of the liquid without the bubbles, and $\mu$ is the viscosity of the liquid without the bubbles. The density equations for the solid and liquid without gas are

$$
\begin{aligned}
& \rho^{(s)}=\rho_{0}^{(s)}\left(1-\beta^{(s)} \sigma\right)=\rho_{0}^{(s)}\left[1+\beta^{(s)} p-\frac{\beta^{(s)} \sigma^{(e f)}}{1-m}\right], \\
& \approx \rho_{0}^{(s)}\left[1+\beta^{(s)} p-\beta^{(s)} \sigma^{(e f)}\right], \\
& \rho^{(L)}=\rho_{0}^{(L)}\left(1+\beta^{(L)} p\right) .
\end{aligned}
$$

The mean density of the gas-liquid mixture is

$$
\rho^{(f)}=(1-\phi) \rho^{(L)}+\phi \rho^{(g)}
$$


where $\phi=(4 \pi / 3) R^{3} n_{0}$.

Here $\sigma$ is the true stress, $\phi$ is the volume gas content and $n_{0}$ is the number density of the bubbles per unit volume. In Eq.(2.5) we can neglect the density of the gas $\rho^{(g)}$ due to the low gas content. The change in $\varphi$ is due to the change in the bubble radius $R$. Then Eq.(2.5) becomes

$$
\rho^{(f)}=\rho_{0}^{L}\left(1+\beta^{(L)} p\right)\left(1-\frac{4 \pi}{3} R_{0}^{3} n_{0}\right)
$$

Similarly to [15], we also assume that the pore pressure $p$ is equal to the pressure in the liquid far from the bubble.

\subsection{Stress-strain relation}

In this section we derive the stress-strain relation for the viscoelastic medium based on the rheological Maxwell-Voigt model, which includes the gas bubble. The model includes two friction elements with viscosities $\mu_{1}$ and $\mu_{2}$, three elastic springs with the elastic moduli $E_{1}, E_{2}$, and $E_{3}$, and three oscillating masses $M_{1}, M_{2}$, and $M_{3}$. The total stress in denoted $\sigma$. We also denote the displacements of the elements of the model by $e$ with respective subscripts as shown in Fig.2. Now we write the second Newton's law for the elements and the kinematic relations

$$
\begin{aligned}
& M_{1} \frac{d^{2} e_{1}}{d t^{2}}+M_{2} \frac{d^{2} e_{2}}{d t^{2}}=\sigma-E_{1} e_{1}-E_{2} e_{2}, \\
& e=e_{2}=e_{1}+e_{3}+e_{4}+e_{5}, \\
& M_{3} \frac{d^{2} e_{3}}{d t^{2}}=E_{1} e_{1}-E_{3} e_{3}, \\
& E_{3} e_{3}=\mu_{2} \frac{d e_{4}}{d t}=\mu_{1} \frac{d e_{5}}{d t} .
\end{aligned}
$$

Equations (2.7) generate the following relation between the stress and strain

$$
\begin{gathered}
{\left[E_{1} E_{3}\left(\frac{1}{\mu_{1}}+\frac{1}{\mu_{2}}\right)\right] \sigma+\left(E_{1}+E_{3}\right) \frac{d \sigma}{d t}+M_{3} \frac{d^{3} \sigma}{d t^{3}}=\left[E_{1} E_{2} E_{3}\left(\frac{1}{\mu_{1}}+\frac{1}{\mu_{2}}\right)\right] e+} \\
+\left[\left(\left(E_{1}+E_{2}\right) E_{3}+E_{1} E_{2}\right)\right] \frac{d e}{d t}+\left[E_{1} E_{3} M_{2}\left(\frac{1}{\mu_{1}}+\frac{1}{\mu_{2}}\right)\right] \frac{d^{2} e}{d t^{2}}+\left[\left(\left(E_{1}+E_{2}\right) M_{3}+\right.\right. \\
\left.\left.+\left(E_{3}+E_{1}\right) M_{2}\right)+E_{3} M_{1}\right] \frac{d^{3} e}{d t^{3}}+\left[\left(M_{1}+M_{2}\right) M_{3}\right] \frac{d^{5} e}{d t^{5}}
\end{gathered}
$$

Generalizing Eq.(2.8) using a similar approach to [9], we get

$$
\sigma^{(e f)}+\eta \sum_{q=1,3} b_{q} \frac{D^{q} \sigma^{(e f)}}{D t^{q}}=E_{2} e+\beta^{(s)} k_{b} p+\eta \sum_{q=1,2,3,5} a_{q} \frac{D^{q} e}{D t^{q}}
$$


where $\sigma^{(e f)}$, is the effective stress, $k_{b}$ is the bulk elastic module of the porous matrix, $\eta=\left(E_{1} E_{3}\left(\frac{1}{\mu_{1}}+\frac{1}{\mu_{2}}\right)\right)^{-1}$ and the coefficients $a_{q}$ and $b_{q}$ are expressed as

$$
\begin{aligned}
& a_{1}=\left[\left(E_{2}+E_{1}\right) E_{3}+E_{1} E_{2}\right], \quad a_{2}=M_{2}, \\
& a_{3}=\left[\left(E_{2}+E_{1}\right) M_{3}+\left(E_{3}+E_{1}\right) M_{2}+E_{3} M_{1}\right], \quad a_{5}=\left[\left(M_{2}+M_{1}\right) M_{3}\right], \\
& b_{1}=\left(E_{3}+E_{1}\right), \quad b_{3}=M_{3} .
\end{aligned}
$$

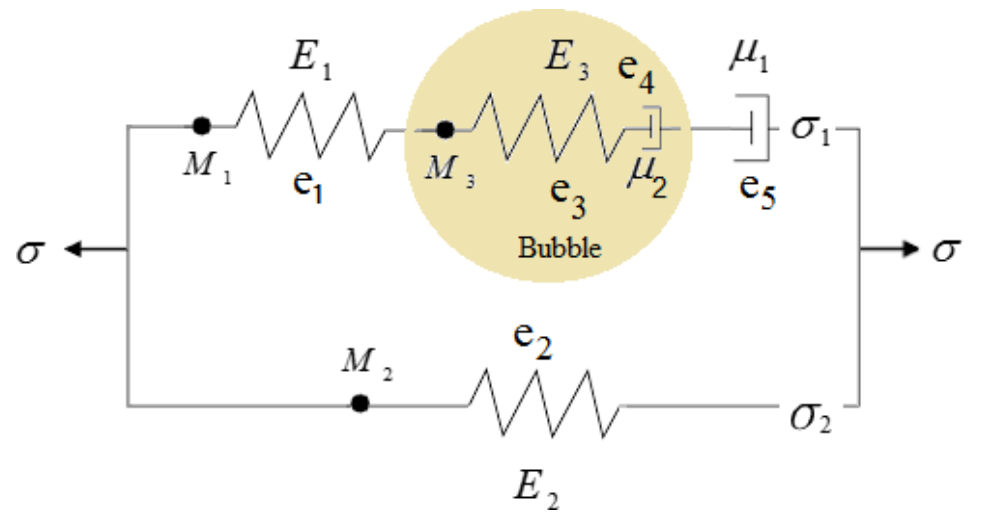

Fig.2. Rheological scheme including a gas bubble.

Finally, we add the closing relation between the deformation $e$ and the velocity $v$ of the solid

$$
\frac{D e}{D t} \equiv \frac{\partial e}{\partial t}+v \frac{\partial e}{\partial x}=\frac{\partial v}{\partial x}
$$

\section{Waves in saturated media including gas bubbles}

Following the approach of Nikolaevskiy [10], we consider the P1-wave in porous media under full saturation. Accordingly, we assume that the mass velocities $v$ and $u$ have the same sign

$$
v=u+O(\varepsilon v)
$$

where $\varepsilon$ is the small parameter. The Darcy force has the order as shown

$$
I=\varepsilon^{\gamma} \delta m(v-u)=\varepsilon^{\gamma} \delta m v, \quad \delta=m \mu / k O(1)
$$

Describing a weakly non-linear wave, we use the running coordinate system with simultaneous scale change

$$
\begin{array}{ll}
\varepsilon=\varepsilon^{\alpha}(x-c t), & \tau=\frac{1}{2} \varepsilon^{\beta} t, \\
\frac{\partial}{\partial x}=\varepsilon^{\alpha} \frac{\partial}{\partial \xi}, & \frac{\partial}{\partial t}=\varepsilon^{\alpha}\left(\frac{1}{2} \varepsilon^{\beta-\alpha} \frac{\partial}{\partial \tau}-c \frac{\partial}{\partial \xi}\right) .
\end{array}
$$


Thus, the constitutive law (2.9) transforms into the following form

$$
\begin{aligned}
& \sigma^{(e f)}+\eta \sum_{q=1,3} b_{q} \varepsilon^{q \alpha}\left(\frac{1}{2} \varepsilon^{\beta-\alpha} \frac{\partial}{\partial \tau}+(v-c) \frac{\partial}{\partial \xi}\right)^{q} \sigma^{(e f)}=E_{2} e+\beta^{(s)} k_{b} p+ \\
& +\eta \sum_{q=1,2,3,5} a_{q} \varepsilon^{q \alpha}\left(\frac{1}{2} \varepsilon^{\beta-\alpha} \frac{\partial}{\partial \tau}+(v-c) \frac{\partial}{\partial \xi}\right)^{q} e .
\end{aligned}
$$

Now, we seek the unknown functions as power series

$$
\begin{aligned}
& v=\varepsilon v_{1}+\varepsilon^{2} v_{2}+\ldots \\
& u=\varepsilon u_{1}+\varepsilon^{2} u_{2}+\ldots \\
& \sigma^{(e f)}=\sigma_{0}^{(e f)}+\varepsilon \sigma_{1}^{(e f)}+\varepsilon^{2} \sigma_{1}^{(e f)}+\ldots \\
& p=p_{0}+\varepsilon p_{1}+\varepsilon^{2} p_{2}+\ldots \\
& m=m_{0}+\varepsilon m_{1}+\varepsilon^{2} m_{2}+\ldots \\
& e=e_{0}+\varepsilon e_{1}+\varepsilon^{2} e_{2}+\ldots \\
& \phi=\phi_{0}+\varepsilon \phi_{1}+\varepsilon^{2} \phi_{2}+\ldots \\
& R=R_{0}\left(1+\varepsilon R_{1}+\varepsilon^{2} R_{2}+\ldots\right)
\end{aligned}
$$

\subsection{The first approximation}

By assuming $\beta=\alpha+1, \alpha=1$ and using Eqs (3.5), we collect the linear terms $\sim \varepsilon$ in system (2.1)

$$
\begin{aligned}
& -\left(1-m_{0}\right) \rho_{0}^{(s)} c \frac{\partial v_{1}}{\partial \xi}=\frac{\partial \sigma_{1}^{(e f)}}{\partial \xi}-\left(1-m_{0}\right) \frac{\partial p_{1}}{\partial \xi}, \\
& -m_{0} \rho_{0}^{(f)} c \frac{\partial u_{1}}{\partial \xi}=-m_{0} \frac{\partial p_{1}}{\partial \xi}, \\
& \rho_{0}^{(s)} c \frac{\partial m_{1}}{\partial \xi}-\left(1-m_{0}\right) c \frac{\partial \rho_{1}^{(s)}}{\partial \xi}+\left(1-m_{0}\right) \rho_{0}^{(s)} \frac{\partial v_{1}}{\partial \xi}=-\frac{1}{2}\left(1-m_{0}\right) \frac{\partial \rho_{0}^{(s)}}{\partial \tau}, \\
& -m_{0} c \frac{\partial \rho_{1}^{(f)}}{\partial \xi}-\rho_{0}^{(f)} c \frac{\partial m_{1}}{\partial \xi}+m_{0} \rho_{0}^{(f)} \frac{\partial u_{1}}{\partial \xi}=-\frac{1}{2} m_{0} \frac{\partial \rho_{0}^{(f)}}{\partial \tau} .
\end{aligned}
$$


The system (3.6) gives the integrals

$$
\begin{aligned}
& \left(1-m_{0}\right) \rho_{0}^{(s)} c v_{1}=-\sigma_{1}^{(e f)}+\left(1-m_{0}\right) p_{1}, \\
& m_{0} \rho_{0}^{(f)} c u_{1}=m_{0} p_{1}, \\
& \left(1-m_{0}\right) \rho_{0}^{(s)} v_{1}=\left(\left(1-m_{0}\right) \rho_{1}^{(s)}-\rho_{0}^{(s)} m_{1}\right) c \\
& m_{0} \rho_{0}^{(f)} u_{1}=\left(\rho_{0}^{(f)} m_{1}+m_{0} \rho_{l}^{(f)}\right) c .
\end{aligned}
$$

According to Eqs (2.3) and (2.6) the terms $\sim \varepsilon$ in the density series are

$$
\begin{aligned}
& \rho_{1}^{(s)}=\rho_{0}^{(s)}\left(\beta^{(s)} p_{1}-\frac{\beta^{(s)} \sigma_{1}^{(e f)}}{\left(1-m_{0}\right)}\right), \\
& \rho_{1}^{(f)}=\rho_{0}^{(L)}\left(\beta^{(L)} \kappa_{l} p_{1}-4 \pi n_{0} \kappa_{2} R_{0}^{3} R_{l}\right),
\end{aligned}
$$

and also

$$
\rho_{0}^{(f)}=\kappa_{1} \kappa_{2} \rho_{0}^{(L)}
$$

where

$$
\kappa_{1}=1-\frac{4 \pi}{3} R_{0}^{3} n_{0}, \quad \kappa_{2}=1+\beta^{(L)} p
$$

Inserting Eqs (3.8) and (3.9) into the last two Eqs in (3.7) (mass equations) we get

$$
\begin{aligned}
& \left(1-m_{0}\right) v_{1}=\left[\left(1-m_{0}\right) \beta^{(s)} p_{1}-\beta^{(s)} \sigma_{1}^{(e f)}-m_{1}\right] c, \\
& m_{0} u_{1}=\left[m_{1}+\frac{m_{0} \beta^{(L)} p_{1}}{\kappa_{2}}-\frac{4 \pi n_{0} m_{0} R_{0}^{3} R_{1}}{\kappa_{1}}\right] .
\end{aligned}
$$

The combination of Eqs (3.10) and (3.11) gives

$$
\left(1-m_{0}\right) v_{1}+m_{0} u_{1}=\left[\frac{\left(\beta+\left(1-m_{0}\right) \beta^{(s)} \beta^{(L)} p_{0}\right) p_{1}}{\kappa_{2}}-\beta^{(s)} \sigma_{1}^{(e f)}-\frac{4 \pi n_{0} m_{0} R_{0}^{3} R_{1}}{\kappa_{1}}\right] c .
$$

The condition (3.1) means $v_{l}=u_{1}$, therefore Eq.(3.12) becomes

$$
v_{1}=\left[\frac{\left(\beta+\left(1-m_{0}\right) \beta^{(s)} \beta^{(L)} p_{0}\right) p_{1}}{\kappa_{2}}-\beta^{(s)} \sigma_{1}^{\left({ }^{(e f)}\right.}-\frac{4 \pi n_{0} m_{0} R_{0}^{3} R_{1}}{\kappa_{1}}\right] c .
$$

Due to the conditions $v_{1}=u_{1}, \rho_{0}=\left(1-m_{0}\right) \rho_{0}^{(s)}+m_{0} \rho_{0}^{(L)}$ and using Eq.(3.9), the first two of the momentum Eqs (3.7) give 


$$
\rho_{0} c v_{1}=-\sigma_{1}^{(e f)}+A p_{1}
$$

where $A=\left(1-m_{0}\right)+\frac{m_{0}}{\kappa_{1} \kappa_{2}}$. Now, the linear terms $\sim \varepsilon$ in relations (2.10) and (3.4) give

$$
\begin{aligned}
& \frac{1}{2} \frac{\partial e_{0}}{\partial \tau}-c \frac{\partial e_{1}}{\partial \xi}+v_{1} \frac{\partial e_{0}}{\partial \xi}=\frac{\partial v_{1}}{\partial \xi} \\
& \sigma_{1}^{(e f)}-E_{2} e_{1}-\beta^{(s)} k_{b} p_{1}=T
\end{aligned}
$$

where $\quad T \equiv \eta\left[\sum_{q=1,2,3,5} a_{q}(-c)^{q} \varepsilon^{q-1} \frac{\partial^{q} e_{0}}{\partial \xi^{q}}+\sum_{q=1,3} b_{q} c^{q} \varepsilon^{q-1} \frac{\partial^{q} \sigma_{0}^{(e f)}}{\partial \xi^{q}}\right]$.

The linear terms $\sim \varepsilon$ in the bubble Eq.(2.2) give

$$
-\frac{\mu c}{\rho_{0}^{(L)} \kappa_{2}}\left[\frac{4}{R_{0}}+\frac{m_{0} R_{0}}{k}\right] \frac{\partial R_{0}}{\partial \xi}=-\frac{1}{\rho_{0}^{(L)} \kappa_{2}}\left(p_{0} \chi R_{1}+p_{1}\right) .
$$

Equations (3.15), (3.16) and (3.17) lead to the integrals

$$
e_{1}=-\frac{v_{1}}{c}, \quad \sigma_{1}^{(e f)}=E_{2} e_{1}+\beta^{(s)} k_{b} p_{1}, \quad p_{1}=-p_{0} \chi R_{1} .
$$

The effective stress $\sigma_{l}^{(e f)}$ in Eq.(3.18) can be rewritten as

$$
\sigma_{1}^{(e f)}=-\left[\frac{E_{2} v_{1}}{c}+p_{0} \chi \beta^{(s)} k_{b} R_{1}\right]
$$

Substituting (3.19) and the value of $p_{1}$ from Eq.(3.18) into (3.13), leads to

$$
\left(1-\beta^{(s)} E_{2}\right) v_{1}+\left(B-\beta^{(s)} \beta^{(s)} k_{b}\right) p_{0} \chi R_{1} c=0,
$$

where

$$
B=\frac{\left(\beta+\left(1-m_{0}\right) \beta^{(s)} \beta^{(L)} p_{0}\right)}{\kappa_{2}}+\frac{4 \pi n_{0} m_{0} R_{0}^{3}}{\kappa_{1} p_{0} \chi} .
$$

Now, from Eq.(3.14) and using the value of $p_{1}$ from Eq.(3.18), we obtain the effective stress as

$$
\sigma_{1}^{(e f)}=-\left(p_{0} c v_{1}+A\right) p_{0} \chi R_{1}
$$

The combination of Eqs (3.19) and (3.21) results in

$$
\left(E_{2}-p_{0} c^{2}\right) v_{l}-\left(A-\beta^{(s)} k_{b}\right) p_{0} \chi R_{l} c=0
$$


Equations (3.20) and (3.22) must coincide, therefore

$$
\left|\begin{array}{ll}
\left(1-\beta^{(s)} E_{2}\right) & \left(B-\beta^{(s)} \beta^{(s)} k_{b}\right) p_{0} \chi \\
\left(E_{2}-p_{0} c^{2}\right) & \left(A-\beta^{(s)} k_{b}\right) p_{0} \chi
\end{array}\right|=0 .
$$

Equation (3.23) gives the velocity of the wave

$$
c^{2}=\frac{\left(A-\beta^{(s)} k_{b}\right) Z+E_{2}}{\rho_{0}}
$$

where

$$
Z=\frac{\left(1-\beta^{(s)} E_{2}\right)}{B-\beta^{(s)} \beta^{(s)} k_{b}}
$$

Thus, all the variables are expressed through any one selected variable, for example, the velocity $v_{1}$

$$
\begin{aligned}
& e_{1}=-\frac{v_{1}}{c}, \quad \sigma_{1}^{(e f)}=-\left(E_{2}-\beta^{(s)} k_{b} Z\right) \frac{v_{1}}{c}, \quad p_{1}=Z \frac{v_{1}}{c}, \\
& R_{1}=-\frac{Z}{p_{0} \chi} \frac{v_{1}}{c}, \quad m_{1}=\left[\left(\left(1-m_{0}\right)-\beta^{(s)} k_{b}\right) \beta^{(s)} Z+\beta^{(s)} E_{2}-\left(1-m_{0}\right)\right] \frac{v_{1}}{c}, \\
& \rho_{1}^{(f)}=\rho_{0}^{(L)} Z\left(\beta^{(L)} \kappa_{1}+\frac{4 \pi n_{0} \kappa_{2} R_{0}^{3}}{p_{0} \chi}\right) \frac{v_{1}}{c}, \quad \rho_{1}^{(s)}=\rho_{0}^{(s)} \beta^{(s)}\left(Z\left(1-\beta^{(s)} k_{b}\right)+E_{2}\right) \frac{v_{1}}{c} .
\end{aligned}
$$

\subsection{The second approximation}

Collecting the quadratic terms $\sim \varepsilon^{2}$ in Eq.(3.4), we get

$$
\sigma_{2}^{(e f)}-E_{2} e_{2}-\beta^{(s)} k_{b} p_{2}=T
$$

where $\quad T \equiv \eta\left[\sum_{q=1,2,3,5} a_{q}(-c)^{q} \varepsilon^{q-1} \frac{\partial^{q} e_{1}}{\partial \xi^{q}}+\sum_{q=1,3} b_{q} c^{q} \varepsilon^{q-1} \frac{\partial^{q} \sigma_{l}^{(e f)}}{\partial \xi^{q}}\right]$.

Note that here we keep (as Nikolaevskiy did in [10]) higher powers of $\varepsilon$ to represent small corrections to the leading terms. These corrections will eventually translate into small corrections in the Nikolaevskiy equation derived further in this paper; they will be the object of our study. Thus

$$
\frac{\partial \sigma_{2}^{(e f)}}{\partial \xi}-E_{2} \frac{\partial e_{2}}{\partial \xi}-\beta^{(s)} k_{b} \frac{\partial p_{2}}{\partial \xi}=\frac{\partial T}{\partial \xi} .
$$

From Eq.(2.10) in the order $\sim \varepsilon^{2}$, we get 


$$
\begin{aligned}
& \frac{\partial}{\partial \xi}\left(c e_{2}+v_{2}\right)=F, \\
& F=-\frac{1}{2}\left(\frac{1}{2} \frac{\partial v_{1}}{\partial \tau}+\frac{\partial v_{1} v_{1}}{\partial \xi}\right)
\end{aligned}
$$

Therefore

$$
\frac{\partial e_{2}}{\partial \xi}=\frac{F}{c}-\frac{1}{c} \frac{\partial v_{2}}{\partial \xi}
$$

Substituting Eq.(3.29) into Eq.(3.27), we obtain

$$
\frac{\partial}{\partial \xi}\left(c \sigma_{2}^{(e f)}+E_{2} v_{2}-c \beta^{(s)} k_{b} p_{2}\right)=E_{2} F+c \frac{\partial T}{\partial \xi} .
$$

From the momentum Eq.(2.1) for the solid and liquid, we get

$$
\left(1-m_{0}\right) \rho_{0}^{(s)} c \frac{\partial v_{2}}{\partial \xi}+\frac{\partial \sigma_{2}^{(e f)}}{\partial \xi}-\left(1-m_{0}\right) \frac{\partial p_{2}}{\partial \xi}=\Sigma_{1}
$$

where

$$
\Sigma_{1}=\left(1-m_{0}\right) \rho_{0}^{(s)}\left(\frac{1}{2} \frac{\partial v_{1}}{\partial \tau}+\frac{\partial v_{1} v_{1}}{\partial \xi}\right)-\left(1-m_{0}\right) \rho_{1}^{(s)} c \frac{\partial v_{1}}{\partial \xi}+m_{l} \rho_{0}^{(s)} c \frac{\partial v_{1}}{\partial \xi}-m_{1} \frac{\partial p_{1}}{\partial \xi}+\varepsilon^{\gamma-1} \delta m_{0} v_{1}
$$

and

where

$$
m_{0} \rho_{0}^{(f)} c \frac{\partial u_{2}}{\partial \xi}-m_{0} \frac{\partial p_{2}}{\partial \xi}=\Sigma_{2}
$$

$$
\Sigma_{2}=m_{0} \rho_{0}^{(f)}\left(\frac{1}{2} \frac{\partial u_{1}}{\partial \tau}+\frac{\partial u_{1} u_{1}}{\partial \xi}\right)-m_{0} \rho_{1}^{(f)} c \frac{\partial u_{1}}{\partial \xi}-m_{1} \rho_{0}^{(f)} c \frac{\partial u_{1}}{\partial \xi}+m_{1} \frac{\partial p_{1}}{\partial \xi}-\varepsilon^{\gamma-1} \delta m_{0} u_{1} .
$$

Due to condition (3.1), the combination of Eq.(3.31) with (3.32) give

$$
\rho_{0} c \frac{\partial v_{2}}{\partial \xi}+\frac{\partial \sigma_{2}^{(e f)}}{\partial \xi}-\frac{\partial p_{2}}{\partial \xi}=\Sigma
$$

where $\sum=\Sigma_{1}+\sum_{2}$, so that

$$
\begin{aligned}
& \sum=\rho_{0}\left(\frac{1}{2} \frac{\partial v_{1}}{\partial \tau}+\frac{\partial v_{1} v_{1}}{\partial \xi}\right)-c\left(\left(1-m_{0}\right) \rho_{0}^{(s)} \beta^{(s)}+m_{0} \rho_{0}^{(L)} \beta^{(L)} \kappa_{1}\right) \frac{\partial p_{1} v_{1}}{\partial \xi}+c \rho_{0}^{(s)} \beta^{(s)} \frac{\partial \sigma_{1}^{(e f)} v_{1}}{\partial \xi}+ \\
& +c \rho_{0}^{(L)} 4 \pi n_{0} m_{0} \kappa_{2} R_{0}^{3} \frac{\partial R_{1} v_{1}}{\partial \xi}+c\left(\rho_{0}^{(s)}-\kappa_{1} \kappa_{2} \rho_{0}^{(L)}\right) \frac{\partial m_{1} v_{1}}{\partial \xi} .
\end{aligned}
$$

Equations (3.30) and (3.33) result in

$$
\frac{\partial}{\partial \xi}\left[\left(E_{2}-p_{0} c^{2}\right) v_{2}+\left(1-\beta^{(s)} k_{b}\right) c p_{2}\right]=E_{2} F-c \sum+c \frac{\partial T}{\partial \xi} .
$$


From the bubble Eq.(2.2), in the order $\sim \varepsilon^{2}$,

$$
\begin{aligned}
& -\frac{\mu c}{\rho_{0}^{(L)} \kappa_{2}}\left[4+\frac{m_{0} R_{0}^{2}}{k}\right] \frac{\partial R_{1}}{\partial \xi}=\frac{1}{\rho_{0}^{(L)} \kappa_{2}}\left[\frac{\beta^{(L)} p_{0} \chi}{\kappa_{2}} p_{1} R_{1}+\frac{\beta^{(L)}}{\kappa_{2}} p_{1}^{2}+\right. \\
& \left.+\frac{p_{0} \chi(\chi+1)}{2} R_{1}^{2}-p_{0} \chi R_{2}-p_{2}\right] .
\end{aligned}
$$

We re-write Eq.(3.35) as

$$
p_{2}=\Gamma-p_{0} \chi R_{2}
$$

where $\quad \Gamma=\mu c\left(4+\frac{m_{0} R_{0}^{2}}{k}\right) \frac{\partial R_{1}}{\partial \xi}+\frac{\beta^{(L)} p_{1}}{\kappa_{2}}\left(p_{0} \chi R_{1}+p_{1}\right)+\frac{p_{0} \chi(\chi+1)}{2} R_{1}^{2}$.

Now we substitute the value of $p_{2}$ from Eq.(3.36) into Eq.(3.34) to get

$$
\begin{aligned}
& \frac{\partial}{\partial \xi}\left[\left(E_{2}-p_{0} c^{2}\right) v_{2}-\left(1-\beta^{(s)} k_{b}\right) p_{0} \chi R_{2} c\right]=E_{2} F-c \sum+ \\
& +c \frac{\partial T}{\partial \xi}-c\left(1-\beta^{(s)} k_{b}\right) \frac{\partial \Gamma}{\partial \xi} .
\end{aligned}
$$

In the second order, the mass balances (2.1) for the solid and liquid-gas mixture have the form

$$
\begin{aligned}
& \frac{\partial}{\partial \xi}\left(\left(1-m_{0}\right) v_{2}-\left[\left(1-m_{0}\right) \beta^{(s)} p_{2}-\beta^{(s)} \sigma_{2}^{(e f)}-m_{2}\right] c\right)=\Lambda^{(s)} / \rho_{0}^{(s)} \\
& \frac{\partial}{\partial \xi}\left(m_{0} u_{2}-\left[m_{2}+\frac{m_{0} \beta^{(L)} p_{2}}{\kappa_{2}}-\frac{4 \pi n_{0} m_{0} R_{0}^{3}\left(R_{2}+R_{1}^{2}\right)}{\kappa_{1}}\right.\right. \\
& \left.\left.\frac{4 \pi n_{0} m_{0} R_{0}^{3} p_{0} \chi \beta^{(L)} R_{1}^{2}}{\kappa_{1} \kappa_{2}}\right] c\right)=\Lambda^{(L)} / \rho_{0}^{(L)}
\end{aligned}
$$

where

$$
\begin{aligned}
& \Lambda^{(s)}=\rho_{0}^{(s)} \frac{1}{2} \frac{\partial}{\partial \tau}\left[\left(m_{1}-\left(1-m_{0}\right) \beta^{(s)} p_{1}+\beta^{(s)} \sigma_{2}^{(e f)}\right)\right]+ \\
& +\rho_{0}^{(s)} \frac{\partial}{\partial \xi}\left[m_{l} v_{1}-\left(\left(1-m_{0}\right) p_{1}+\sigma_{l}^{(e f)}\right) \beta^{(s)} v_{l}-c \beta^{(s)} m_{l}\left(p_{1}-\frac{\sigma_{l}^{(e f)}}{\left(1-m_{0}\right)}\right)\right], \\
& \Lambda^{(L)}=-\rho_{0}^{(L)} \frac{1}{2} \frac{\partial}{\partial \tau}\left[\kappa_{1}\left(m_{1} \kappa_{2}+m_{0} \beta^{(L)} p_{1}\right)-4 \pi n_{0} \kappa_{2} R_{0}^{3} R_{1}\right]+ \\
& +\rho_{0}^{(L)} \frac{\partial}{\partial \xi}\left[\left(\beta^{(L)} \kappa_{1} p_{1}-4 \pi n_{0} \kappa_{2} R_{0}^{3} R_{1}\right)\left(c m_{1}-m_{0} u_{1}\right)\right]-\kappa_{1} \kappa_{2} \rho_{0}^{(L)} \frac{\partial m_{1} u_{1}}{\partial \xi} .
\end{aligned}
$$

The combination of Eqs (3.38) and (3.39) gives 


$$
\begin{aligned}
& \frac{\partial}{\partial \xi}\left(v_{2}-\left[\frac{\left(\beta+\left(1-m_{0}\right) \beta^{(s)} \beta^{(L)} p_{0}\right) p_{2}}{\kappa_{2}}-\beta^{(s)} \sigma_{2}^{(e f)}-\frac{4 \pi n_{0} m_{0} R_{0}^{3}\left(R_{2}+R_{1}^{2}\right)}{\kappa_{1}}+\right.\right. \\
& \left.\left.+\frac{4 \pi n_{0} m_{0} R_{0}^{3} p_{0} \chi \beta^{(L)} R_{1}^{2}}{\kappa_{1} \kappa_{2}}\right] c\right)=\Lambda,
\end{aligned}
$$

where

$$
\Lambda=\Lambda^{(s)} / \rho_{0}^{(s)}+\Lambda^{(L)} / \rho_{0}^{(L)}
$$

From Eq.(3.30) we have

$$
\frac{\partial \sigma_{2}^{(e f)}}{\partial \xi}=\frac{\partial}{\partial \xi}\left(T+\beta^{(s)} k_{b} \Gamma-\beta^{(s)} k_{b} p_{0} \chi R_{2}-\frac{E_{2}}{c} v_{2}\right)+\frac{1}{c} E_{2} F
$$

Now we insert Eq.(3.43) and the value of $p_{2}$ represented by Eq.(3.36) into Eq.(3.42)

where

$$
\begin{aligned}
& \frac{\partial}{\partial \xi}\left[\left(1-E_{2} \beta^{(s)}\right) v_{2}-\left(\omega_{1} p_{0} \chi-\frac{4 \pi n_{0} m_{0} R_{0}^{3}}{\kappa_{1}}\right) R_{2} c\right]= \\
& =\Lambda-\beta^{(s)} E_{2} F-c \beta^{(s)} \frac{\partial T}{\partial \xi}-c \omega_{1} \frac{\partial \Gamma}{\partial \xi}+c \omega_{2} \frac{\partial R_{1}^{2}}{\partial \xi}
\end{aligned}
$$

$$
\begin{aligned}
& \omega_{1}=k_{b} \beta^{(s)} \beta^{(s)}+\frac{\left(\beta+\left(1-m_{0}\right) \beta^{(s)} \beta^{(L)} p_{0}\right)}{\kappa_{2}}, \\
& \omega_{2}=\frac{4 \pi n_{0} m_{0} R_{0}^{3} \beta^{(L)} p_{0} \chi}{\kappa_{1} \kappa_{2}}-\frac{4 \pi n_{0} m_{0} R_{0}^{3}}{\kappa_{1}} .
\end{aligned}
$$

The determinant of the left-hand side of the system of Eqs (3.37) and (3.44) coincides with the determinant of Eq.(3.23), which equals zero. A non-zero solution for $v_{2}$ exists only if the following compatibility condition takes place

$$
\left|\begin{array}{ll}
\left(E_{2}-p_{0} c^{2}\right) & \frac{\partial}{\partial \xi}\left(E_{2} F-c \sum+c \frac{\partial T}{\partial \xi}-c\left(1-\beta^{(s)} k_{b}\right) \frac{\partial \Gamma}{\partial \xi}\right) \\
\left(1-\beta^{(s)} E_{2}\right) & \frac{\partial}{\partial \xi}\left(\Lambda-\beta^{(s)} E_{2} F-c \beta^{(s)} \frac{\partial T}{\partial \xi}-c \omega_{1} \frac{\partial \Gamma}{\partial \xi}+c \omega_{2} \frac{\partial R_{1}^{2}}{\partial \xi}\right)
\end{array}\right|=0 .
$$

(see Appendix). This is the evolution equation with respect to $v \cong v_{1}$

$$
c M \frac{\partial \Gamma}{\partial \xi}-c N \frac{\partial T}{\partial \xi}+c \omega_{2} \psi \frac{\partial R_{1}^{2}}{\partial \xi}+\Lambda \psi+c \Sigma\left(1-E_{2} \beta^{(s)}\right)-E_{2} F N=0
$$

where

$$
\psi=\left(E_{2}-p_{0} c^{2}\right), \quad M=\left(1-\beta^{(s)} k_{b}\right)\left(1-E_{2} \beta^{(s)}\right)-\omega_{1} \psi, \quad N=\left(1-\beta^{(s)} \rho_{0} c^{2}\right) .
$$


Now, we re-write Eq.(3.46) in terms of $v$ and re-group

$$
\begin{gathered}
\frac{1}{2}\left[Y_{1}+\psi\left(\left(1-\kappa_{1} \kappa_{2}\right) \hat{m}_{1}-E_{2} \beta^{(s)}-\left(Y_{2}+\frac{4 \pi n_{0} R_{0}^{3}}{p_{0} \chi}\right) Z\right)\right] \frac{\partial v}{\partial \tau}+ \\
-\left[N \eta c^{2}\left(a_{1}-b_{1}\left(E_{2}-k_{b} \beta^{(s)} Z\right)\right)+\frac{M Z \mu c^{2}}{p_{0} \chi}\left(4+\frac{m_{0} R_{0}^{2}}{k}\right)\right] \frac{\partial^{2} v}{\partial \xi^{2}}+\varepsilon N \eta c^{3} a_{2} \frac{\partial^{3} v}{\partial \xi^{3}}+ \\
-\varepsilon^{2} N \eta c^{4}\left[a_{3}-b_{3}\left(E_{2}-k_{b} \beta^{(s)} Z\right)\right] \frac{\partial^{4} v}{\partial \xi^{4}}-\varepsilon^{4} N \eta a_{5} c^{6} \frac{\partial^{6} v}{\partial \xi^{6}}-\left[\zeta_{1}+\zeta_{2}\right] \frac{\partial v v}{\partial \xi}=0,
\end{gathered}
$$

where

$$
\begin{gathered}
\hat{m}_{1}=\left(\left(1-m_{0}\right)-\beta^{(s)} k_{b}\right) \beta^{(s)} Z+\beta^{(s)} E_{2}-\left(1-m_{0}\right), \quad Y_{1}=\left(E_{2} N+c^{2} \rho_{0}\left(1-E_{2} \beta^{(s)}\right)\right), \\
Y_{2}=m_{0}\left(\kappa_{1} \beta^{(L)}-\beta^{(s)}\right)+\beta^{(s)}\left(1-\beta^{(s)} k_{b}\right), \\
\zeta_{1}=\psi\left(\hat{m}_{1}-\left(1-m_{0}\right) \beta^{(s)} Z+\beta^{(s)}\left(E_{2}-k_{b} \beta^{(s)} Z\right)\left(1-\hat{m}_{1}\right)-\beta^{(s)} Z \hat{m}_{1}+\kappa_{1} \beta^{(L)} Z \hat{m}_{1}-\kappa_{1} \kappa_{2}+\right. \\
\left.+4 \pi n_{0} \kappa_{2} R_{0}^{3} \frac{Z}{p_{0} \chi}\left(\hat{m}_{1}-m_{0}\right)-m_{0} \kappa_{1} \beta^{(L)} Z+\omega_{2} \frac{Z^{2}}{\left(p_{0} \chi\right)^{2}}\right) \\
\zeta_{2}=c^{2}\left(1-E_{2} \beta^{(s)}\right)\left(\rho_{0}-\rho_{0} \kappa_{1} \beta Z-\rho_{0}^{(s)} \beta^{(s)}\left(E_{2}-k_{b} \beta^{(s)} Z\right)-m_{0} \kappa_{2} \rho^{(L)} \frac{Z}{p_{0} \chi}+\right. \\
\left.+\hat{m}_{1}\left(\rho^{(s)}-\kappa_{1} \kappa_{2} \rho^{(L)}\right)\right)+\frac{M(\chi+l)}{2 p_{0} \chi} Z^{2}+E_{2} N .
\end{gathered}
$$

In short, the evolution Eq.(3.47) can be written as

$$
C_{1} \frac{\partial v}{\partial \tau}-C_{2} \frac{\partial^{2} v}{\partial \xi^{2}}+\varepsilon C_{3} \frac{\partial^{3} v}{\partial \xi^{3}}-\varepsilon^{2} C_{4} \frac{\partial^{4} v}{\partial \xi^{4}}-\varepsilon^{4} C_{6} \frac{\partial^{6} v}{\partial \xi^{6}}-\zeta \frac{\partial v v}{\partial \xi}=0
$$

where

$$
\begin{aligned}
& C_{1}=\frac{1}{2}\left[Y_{1}+\psi\left(\left(1-\kappa_{1} \kappa_{2}\right) \hat{m}_{1}-E_{2} \beta^{(s)}-\left(Y_{2}+\frac{4 \pi n_{0} R_{0}^{3}}{p_{0} \chi}\right) Z\right)\right], \\
& C_{2}=\left[N \eta c^{2}\left(a_{1}-b_{1}\left(E_{2}-k_{b} \beta^{(s)} Z\right)\right)+\frac{M Z \mu c^{2}}{p_{0} \chi}\left(4+\frac{m_{0} R_{0}^{2}}{k}\right)\right], \quad C_{3}=N \eta c^{3} a_{2}, \\
& C_{4}=N \eta c^{4}\left(a_{3}-b_{3}\left(E_{2}-k_{b} \beta^{(s)} Z\right)\right), \quad C_{6}=N \eta a_{5} c^{6}, \quad \zeta=\zeta_{1}+\zeta_{2} .
\end{aligned}
$$

\section{Linearized model}

In this section, we consider the linearized version of the model (3.48). Our particular interest is its dissipative part responsible for decay of the wave. 


\subsection{Evaluation of the parameters and the wave velocity}

From [15]-[21], the values of the parameters are: densities, $\rho^{(L)}=1000 \mathrm{~kg} / \mathrm{m}^{3}$ for water, $\rho^{(g)}=2$ $\mathrm{kg} / \mathrm{m}^{3}$ for gas, $\rho^{(s)}=2500 \mathrm{~kg} / \mathrm{m}^{3}$ for solid, porosity $m_{0}=0.25$; bulk modulus $k_{b}=1.7 \times 10^{9} \mathrm{~Pa}$; compressibility $\beta^{(L)}=2 \times 10^{-9} \mathrm{~Pa}^{-1}$ for water, $\beta^{(g)}=2.4 \times 10^{-6} \mathrm{~Pa}^{-1}$ for gas, $\beta^{(s)}=2 \times 10^{-10} \mathrm{~Pa}^{-1}$ for solid; steady pressure $p_{0}=10^{7} \mathrm{~Pa}$; bubble radius; $R_{0}=5 \times 10^{-5} \mathrm{~m}$ volume gas content $\phi_{0}=10^{-3}$; viscosities $\mu_{1}=10^{-3} \mathrm{~Pa} \cdot \mathrm{s}$ for water, $\mu_{2}=2 \times 10^{-5} \mathrm{~Pa} \cdot \mathrm{s}$ for gas; adiabatic exponent $\varsigma=1.4$, and permeability $k=2 \times 10^{-11} \mathrm{~m}^{2}$. Using the data from [14, 16, 21, 22], the values of the parameters of the rheological scheme in Fig.2 are

and

$$
M_{1}=\rho^{(L)} L_{s}^{2}=10^{-2} \mathrm{~kg} / \mathrm{m}, \quad M_{2}=\rho^{(s)} L_{s}^{2}=0.02 \mathrm{~kg} / \mathrm{m}, \quad M_{3}=\rho^{(g)} L_{s}^{2}=2 \times 10^{-6} \mathrm{~kg} / \mathrm{m}
$$

$$
E_{1}=1 / \beta^{(L)}=4 \times 10^{5} \mathrm{~Pa}, \quad E_{2}=c^{2} \rho_{0}=2 \times 10^{7} \mathrm{~Pa}, \quad E_{3}=3 \chi p_{0}=4 \times 10^{7} \mathrm{~Pa},
$$

where we used, just for the purpose of calculation of $E_{i}$ and $M_{i}$, the typical velocity $c \sim 100 \mathrm{~m} / \mathrm{s}$ and the linear size of the oscillator $L_{s}=0.3 \mathrm{~cm}$ from $[16,23]$. We will also explore the values of $E_{i}$ obtained by a different method, namely by using the formula $c^{2} \rho$ for all three phases, with $\rho$ being the density of the liquid, solid and gas, respectively,

$$
E_{1}=c^{2} \rho^{(L)}=1000 \times 10^{4} \mathrm{~Pa}, \quad E_{2}=c^{2} \rho^{(s)}=2500 \times 10^{4} \mathrm{~Pa}, \quad E_{3}=c^{2} \rho^{(g)}=2 \times 10^{4} \mathrm{~Pa} .
$$

Now we apply the formula for the wave velocity (3.24) to show that it gives reasonable order of magnitude. For the wave with the bubbles (3.24) gives $c \approx 582 \mathrm{~m} / \mathrm{s}$ for both variants (a) and (b). For the wave without the bubbles $\left(n_{0}=0, R_{0}=0\right.$, and $\left.M_{3}=0\right) c \approx 740 \mathrm{~m} / \mathrm{s}$. This illustrates, in line with the previous studies that the bubbles may result in a considerable change of the wave velocity. Furthermore, we will also explore the following values of $E_{i}$ obtained experimentally.

$$
E_{1}=10^{6} \mathrm{~Pa}, \quad E_{2}=10^{9} \mathrm{~Pa}, \quad E_{3}=10^{6} \mathrm{~Pa} .
$$

This results in the wave velocity with the bubbles $c \approx 855 \mathrm{~m} / \mathrm{s}$, and the wave velocity without the bubbles $c \approx 950 \mathrm{~m} / \mathrm{s}$.

\subsection{Dissipation relation}

Analysing the linearized model, we are interested in the influence of the bubbles on the decay rate of the wave. This effect is controlled by even derivatives. Therefore, we truncate the linearized Eq.(3.48) to the form

$$
\frac{\partial v}{\partial \tau}=\frac{C_{2}}{C_{1}} \frac{\partial^{2} v}{\partial \xi^{2}}+\varepsilon^{2} \frac{C_{4}}{C_{1}} \frac{\partial^{4} v}{\partial \xi^{4}}+\varepsilon^{4} \frac{C_{6}}{C_{1}} \frac{\partial^{6} v}{\partial \xi^{6}}
$$

For the Fourier modes $v \sim \exp (\lambda t+i k x)$, we get the dissipation relation

$$
\lambda(k)=-\frac{C_{2}}{C_{1}} k^{2}+\varepsilon^{2} \frac{C_{4}}{C_{1}} k^{4}-\varepsilon^{4} \frac{C_{6}}{C_{1}} k^{6},
$$


where $\lambda$ is the decay rate and $k$ is the wave number.

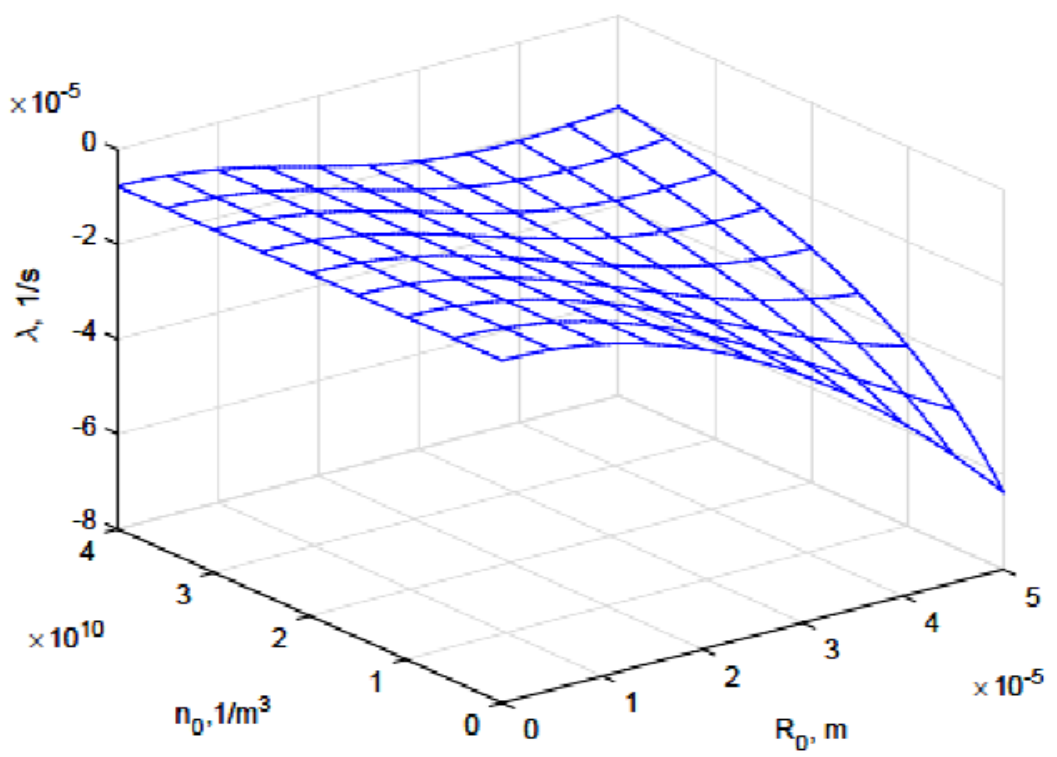

Fig.3. The decay rate for variant (a), $k_{*}=0.251 / \mathrm{m}$.

The plot in Fig.3 shows the decay rate at fixed $k_{*}=0.251 / m$ [9] against $R_{0}$ and $n_{0}$. See that the increase in $R_{0}$ significantly affects the decay rate and makes it in absolute value larger as the bubbles affect the system through the pressure $p_{1}=-p_{0} \chi R_{l}$. As for $n_{0}$, one should disregard the region of small $n_{0}$ in Fig. 3 where the equations of continuum mechanics are not valid. This is because the assumption that each bubble is embedded in its own fluid particle (see Eq.(2.2)) becomes inapplicable due to the large size of the particle.
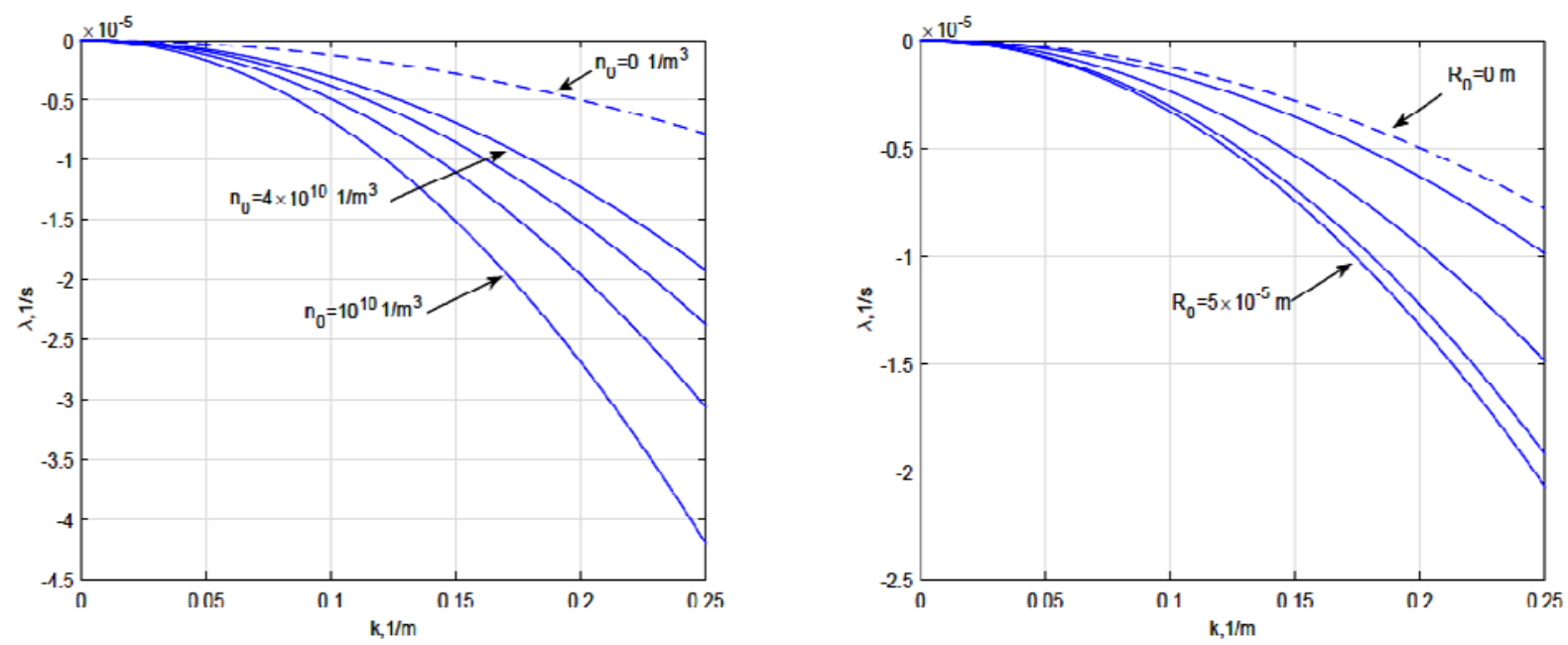

Fig.4. The attenuation curves by formula (4.2) for variant (a). Left: $n_{0}$ varies, $R_{0}=5 \times 10^{-5}$; right: $R_{0}$ varies, $n_{0}=4 \times 10^{10}$. 
Figure 4 compares the attenuation curves of the wave with and without the bubbles. The dashed line describes the case without the bubbles that is $n_{0}=0$, and $R_{0}=0$ and the other lines correspond to the wave with the bubbles. The figure on the left is for varying $n_{0}$ and fixed $R_{0}$. The figure on the right is for varying $R_{0}$ and fixed $n_{0}$. See that the curves lie entirely below zero, which means that the wave decays and the decay rate depends on the number and radius of the bubbles. This result agrees with the conception emphasized in $[24,25]$ about the essentially passive nature of the freely propagating elastic wave. Similar results are obtained for variants (b) and (c) as shown in Figs 5-8.

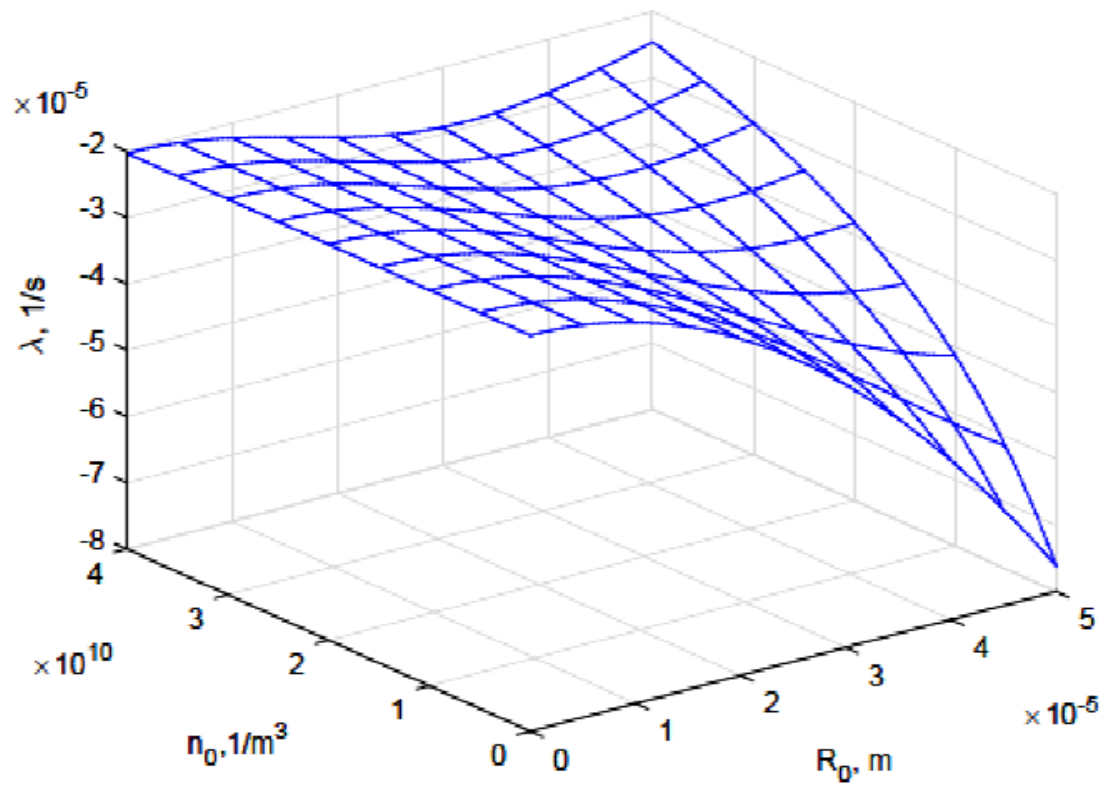

Fig.5. The decay rate for variant (b), $k_{*}=0.251 / \mathrm{m}$.
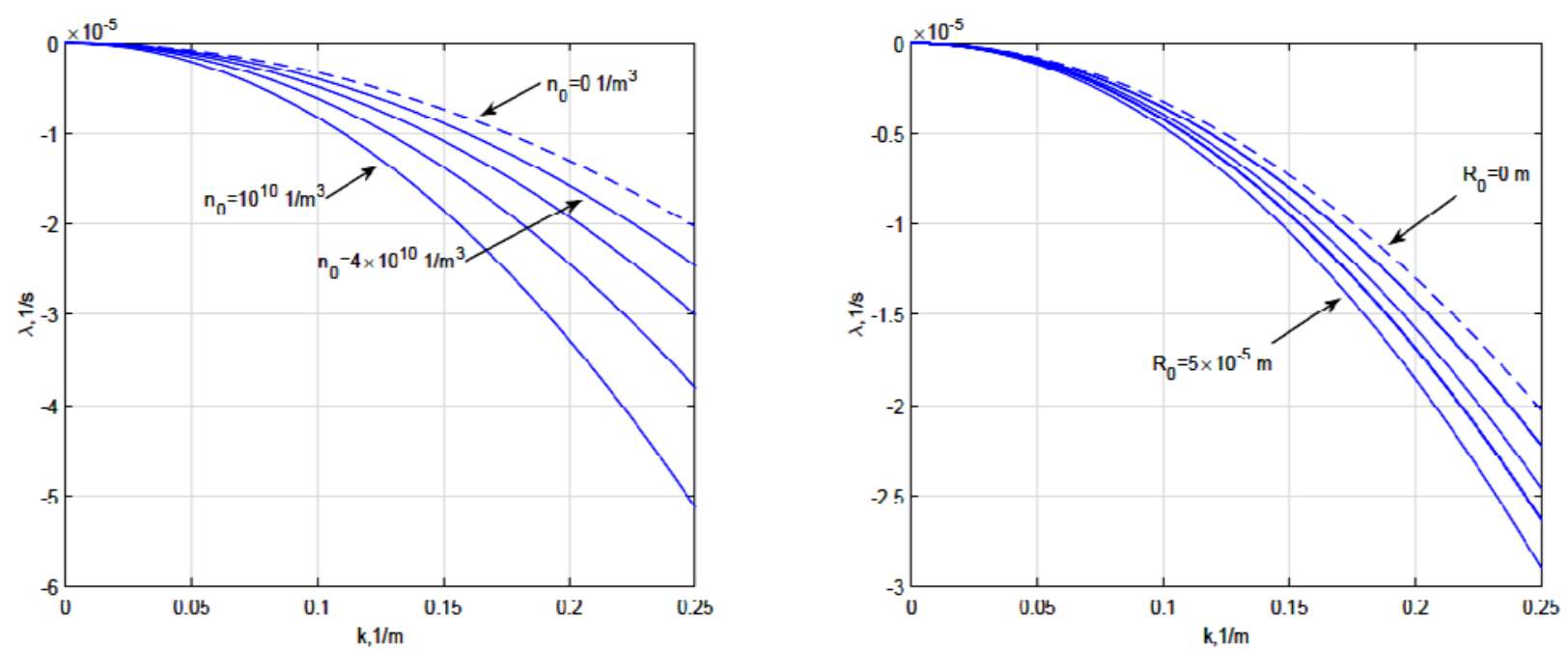

Fig.6. The attenuation curves by formula (4.2) for variant (b). Left: $n_{0}$ varies, $R_{0}=5 \times 10^{-5}$; right: $R_{0}$ varies, $n_{0}=4 \times 10^{10}$. 


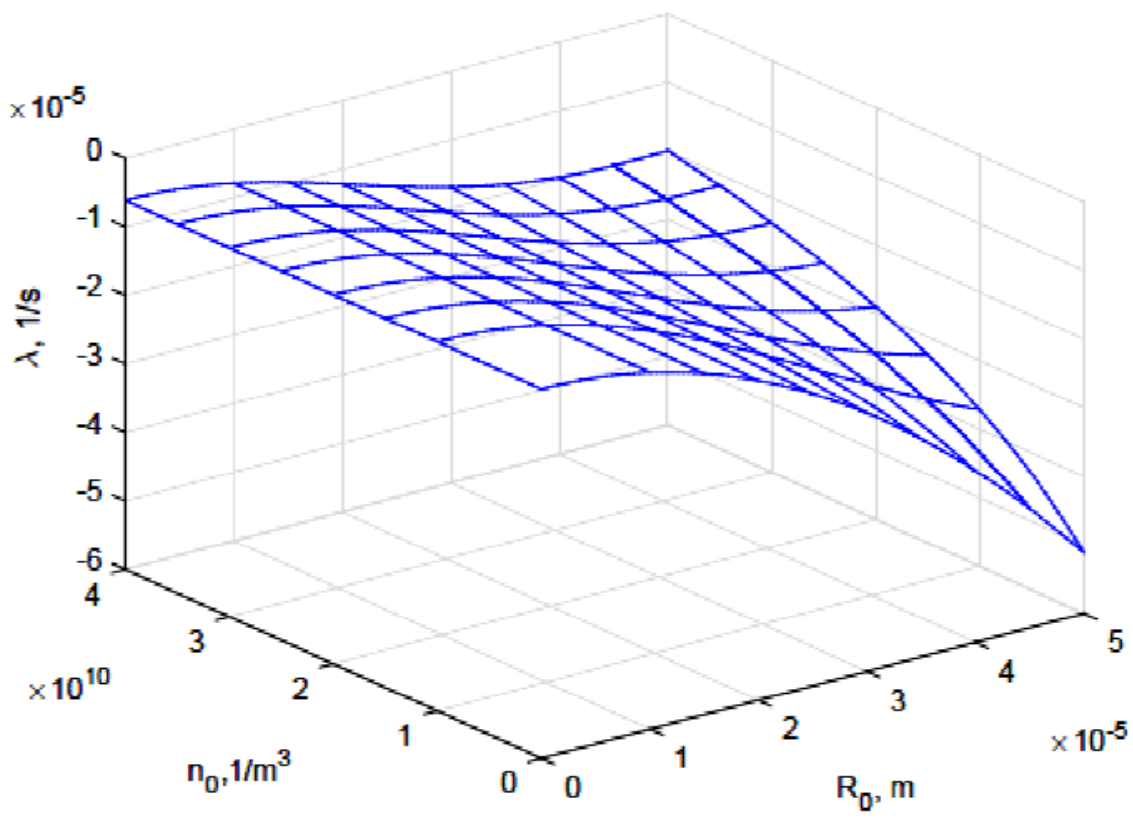

Fig.7. The decay rate for variant (c), $k_{*}=0.251 / \mathrm{m}$.
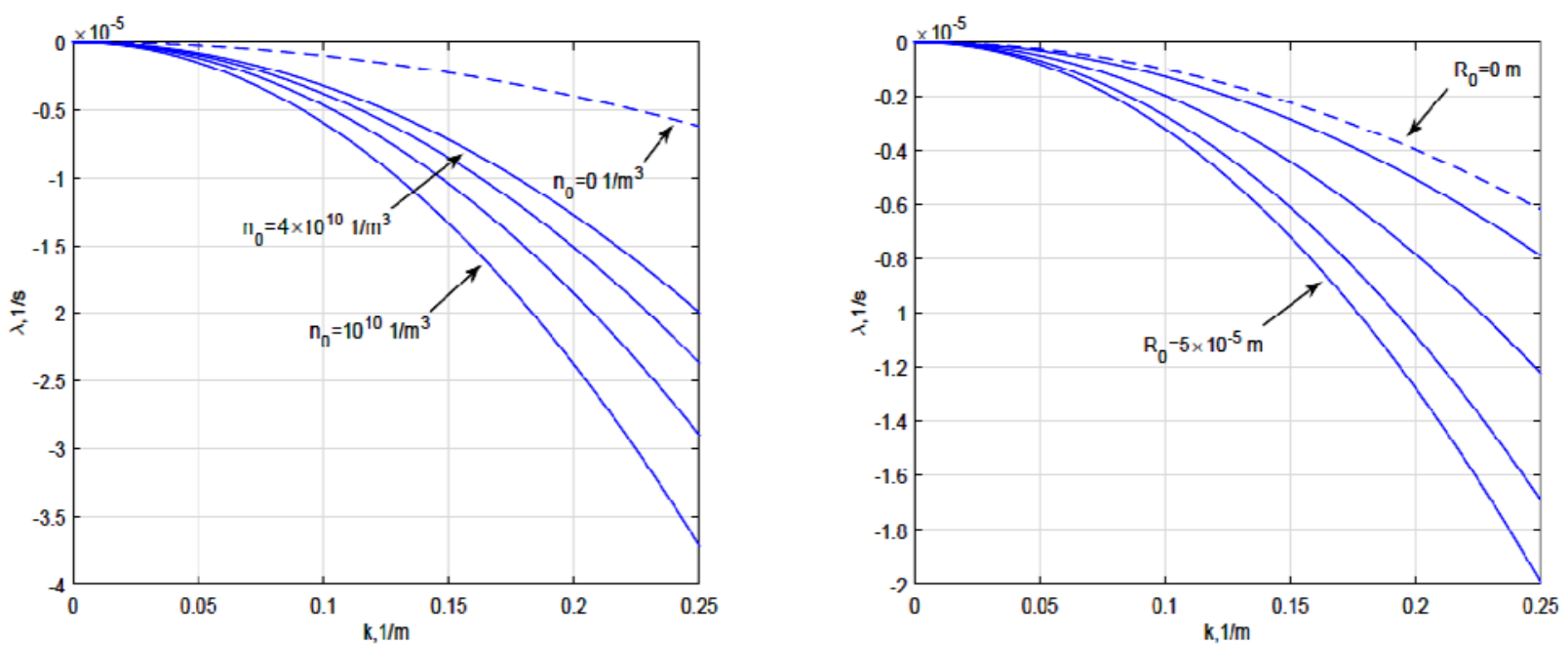

Fig.8. The attenuation curves by formula (4.2) for variant (c). Left: $n_{0}$ varies, $R_{0}=5 \times 10^{-5}$; right: $R_{0}$ varies, $n_{0}=4 \times 10^{10}$.

For a different $k_{*}=0.521 / m[26]$, the results are similar, see Fig.9. 


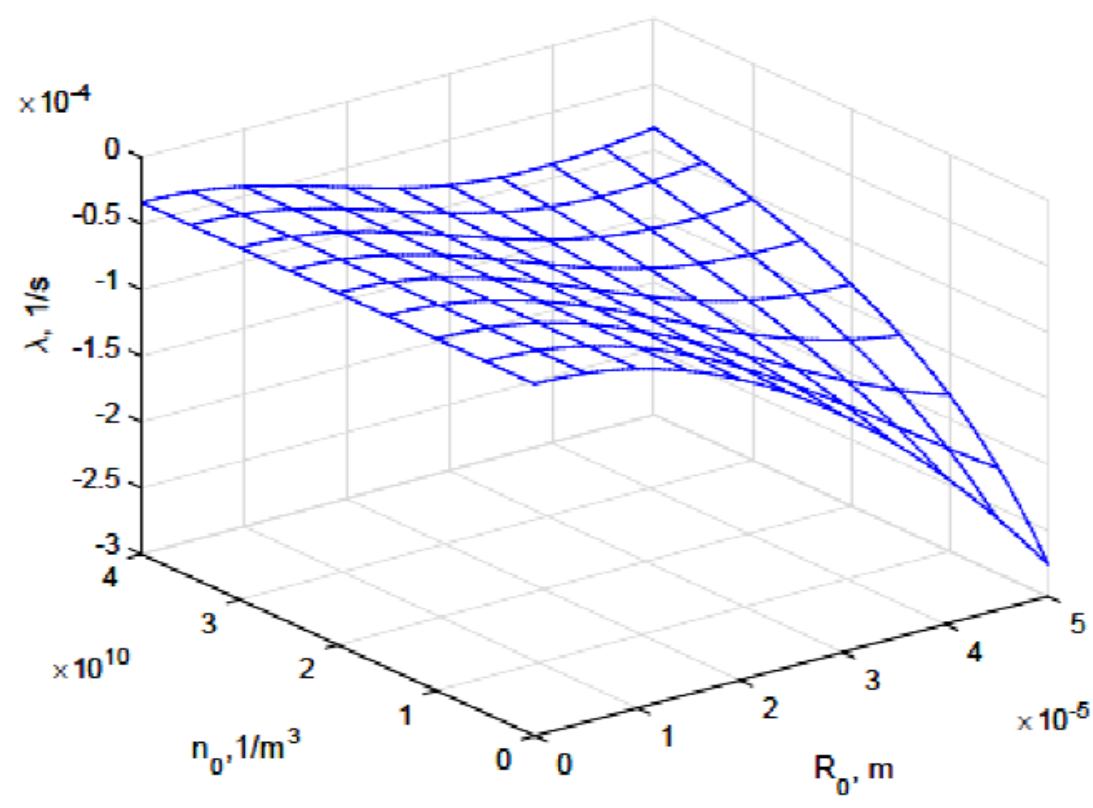

Fig.9. The decay rate for variant (a), $k_{*}=0.521 / \mathrm{m}$.

\section{Conclusions}

We studied the effect of the rheology with gas bubbles and bubble dynamics on the elastic wave in a fluid-saturated medium. The P1 Frenkel-Biot wave is analysed, which corresponds to the fully saturated medium. Using three-segment rheology, we derived the Nikolaevskiy-type equation for the velocity of the solid matrix in the wave. The linearized version of the equation is compared in terms of the decay rate $\lambda(k)$ of the Fourier modes. For both the cases with and without the bubbles, the $\lambda(k)$-curve lies entirely below zero. We discovered that $\lambda(k)$ increases with the increase of the radius of the bubbles but decreases with the increase of the number of the bubbles.

\section{Nomenclature}

$$
\begin{aligned}
k & \text { - permeability, }\left[\mathrm{m}^{2}\right] \\
k_{b} & \text { - bulk modulus, }[\mathrm{Pa}] \\
m & - \text { porosity } \\
p & - \text { pressure, }[\mathrm{Pa}] \\
R & - \text { bubble radius, }[\mathrm{m}] \\
\beta^{(s)} & - \text { compressibility for solid, }\left[\mathrm{Pa}^{-1}\right] \\
\beta^{(L)} & - \text { compressibility for water, }\left[\mathrm{Pa}^{-1}\right] \\
\beta^{(g)} & - \text { compressibility for gas, }\left[\mathrm{Pa}^{-1}\right] \\
\varsigma & - \text { adiabatic exponent } \\
\mu & - \text { viscosity, }[\mathrm{Pa} . \mathrm{s}] \\
\rho^{(s)} & - \text { density for solid, }\left[\mathrm{kg} / \mathrm{m}^{3}\right] \\
\rho^{(L)} & - \text { density for water, }\left[\mathrm{kg} / \mathrm{m}^{3}\right] \\
\rho^{(g)} & - \text { density for gas, }\left[\mathrm{kg} / \mathrm{m}^{3}\right] \\
\phi & - \text { volume gas content }
\end{aligned}
$$




\section{Appendix}

Equation (3.45) can be illustrated with this simple example. We will use the same notations $v_{l}$ and $c$ as in the main text just to resemble the particle velocity and wave velocity

$$
\begin{aligned}
& v_{1}+c v_{1}=0, \\
& 2 v_{1}+4 v_{1}=0 .
\end{aligned}
$$

A non-zero solution of the system exists only if $c=2$ (the eigenvalue of the problem). Here $v_{l}$ is the analogy of the first approximation from our main text. The second approximation, $v_{2}$, satisfies the system

$$
\begin{aligned}
& v_{2}+c v_{2}=f\left[v_{1}\right], \\
& 2 v_{2}+4 v_{2}=g\left[v_{1}\right],
\end{aligned}
$$

which is solvable only if the operators satisfy the equation $g\left[v_{1}\right]=2 f\left[v_{1}\right]$. This is the analogy to the Nikolaevskiy-type equation that we aim to derive.

\section{References}

[1] Biot M.A. (1956): Theory of propagation of elastic waves in a fluid-saturated porous solid. I Low-frequency range. - The Journal of the Acoustical Society of America, vol.28, No.2, pp.168-178.

[2] Biot M.A. (1956): Theory of propagation of elastic waves in a fluid-saturated porous solid. II Higher frequency range. - The Journal of the Acoustical Society of America, vol.28, No.2, pp.179-191.

[3] Frenkel J. (2005): On the theory of seismic and seismoelectric phenomena in a moist soil. - Journal of Engineering Mechanics, vol.131, No.9, pp.879-887.

[4] Biot M.A. (1962): Mechanics of deformation and acoustic propagation in porous media. - Journal of Applied Physics, vol.33, No.4, pp.1482-1498.

[5] Biot M.A. (1962): Generalized theory of acoustic propagation in porous dissipative media. - The Journal of the Acoustical Society of America, vol.34, No.9A, pp.1254-1264.

[6] Klimushin R.R. and Shalashov G.M. (1990): Nonlinear deformation of saturated porous media in the FrenkelBiot Model. - Solid Earth Phys., vol.3.

[7] Plona T.J. (1980): Observation of a second bulk compressional wave in a porous medium at ultrasonic frequencies. - Applied Physics Letters, vol.36, No.4, pp.259-261.

[8] Yang L., Yang D. and Nie J. (2014): Wave dispersion and attenuation in viscoelastic isotropic media containing multiphase flow and its application. - Science China Physics, Mechanics and Astronomy, vol.57, No.6, pp.10681077.

[9] Nikolaevskii V.N. (1989): Dynamics of viscoelastic media with internal oscillations. - S.L. Koh et al. (eds.), Recent Advances in Engineering Science, Springer-Verlag, Berlin, pp.210-221.

[10] Nikolaevskiy V.N. (2008): Non-linear evolution of P-waves in viscous-elastic granular saturated media. Transport in Porous Media, vol.73, No.2, pp.125-140.

[11] Dunin S.Z. and Nikolaevskii V.N. (2005): Nonlinear waves in porous media saturated with live oil. - Acoustical Physics, vol.51, No.1, pp.S61-S66.

[12] Van Wijngaarden L. (1968): On the equations of motion for mixtures of liquid and gas bubbles. - Journal of Fluid Mechanics, vol.33, No.3, pp.465-474. 
[13] Anderson A.L. and Hampton L.D. (1980): Acoustics of gas-bearing sediments I. Back- ground. - The Journal of the Acoustical Society of America, vol.67, No.6, pp.1865-1889.

[14] Nikolaevskiy V.N. and Strunin D.V. (2012): The role of natural gases in seismic of hydrocarbon reservoirs. Elastic Wave Effect on Fluid in Porous Media, Proceedings 2012, pp.25-29.

[15] Dunin S.Z., Mikhailov D.N. and Nikolayevskii V.N. (2006): Longitudinal waves in partially saturated porous media: the effect of gas bubbles. - Journal of Applied Mathematics and Mechanics, vol.70, No.2, pp.251-263.

[16] Nikolaevskii V.N. (1985): Viscoelasticity with internal oscillators as a possible model of seismoactive medium. Doklady Akademii Nauk SSSR, vol.283, No.6, pp.1321-1324.

[17] Sutton G.P. and Oscar B. (2016): Rocket propulsion elements. - John Wiley and Sons.

[18] Carcione J.M. (1998): Viscoelastic effective rheologies for modelling wave propagation in porous media. Geophysical Prospecting, vol.46, No.3, pp.249-270.

[19] Mikhailov D.N. (2010): The influence of gas saturation and pore pressure on the characteristics of the FrenkelBiot $P$ waves in partially saturated porous media. - Izvestiya, Physics of the Solid Earth, vol.46, No.10, pp.897909.

[20] Smeulders D.M. (2005): Experimental evidence for slow compressional waves. - Journal of Engineering Mechanics, vol.131, No.9, pp.908-917.

[21] Nikolaevskiy V.N. (2016): A real P-wave and its dependence on the presence of gas. - Izvestiya, Physics of the Solid Earth, vol.52, No.1, pp.1-13.

[22] Nikolaevskii V.N. and Stepanova G.S. (2005): Nonlinear seismic and the acoustic action on the oil recovery from an oil pool. - Acoustical Physics, vol.51, No.1, pp.S131-S139.

[23] Vilchinska N., Nikolajevskiy V.N. and Lisin V. Slow waves and natural oscillations in sandy marine soils. Izvestiya Acad. Nauk SSSR, Oceanology 4.

[24] Strunin D.V. (2014): On dissipative nature of elastic waves. - Journal of Coupled Systems and Multiscale Dynamics, vol.2, No.2, pp.70-73.

[25] Strunin D.V. and Ali A.A. (2016): On nonlinear dynamics of neutral modes in elastic waves in granular media. Journal of Coupled Systems and Multiscale Dynamics, vol.4, No.3, pp.163-169.

[26] Beresnev I.A. and Nikolaevskiy V.N. (1993): A model for nonlinear seismic waves in a medium with instability. Physica D: Nonlinear Phenomena, vol.66, No.1-2, pp.1-6.

Received: September 22, 2017

Revised: April 16, 2018 